\title{
Artículos
}

\section{Outsourcing en las unidades de información de las organizaciones}

\author{
Por Pablo Lara Navarra y José Ángel Martínez Usero
}

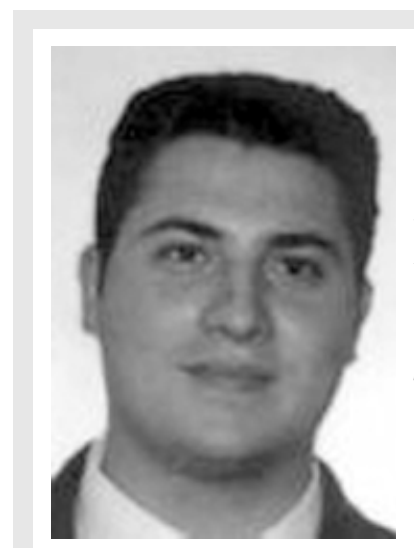

Pablo Lara Navarra, diplomado en biblioteconomía y documentación y licenciado en documentación, es profesor coordinador del área de gestión de la información de la UOC, la universidad virtual. Actualmente desarrolla proyectos de visibilidad de información en contenidos educativos on-line y gestión de recursos electrónicos en bibliotecas virtuales.

\section{ganisations}

Resumen: El outsourcing como herramienta de gestión empresarial aporta al campo de la información y documentación nuevas formas de gestionar las unidades de información en las organizaciones. Adentrarnos en el outsourcing implica conocer con profundidad los entresijos del concepto, modalidades de externalización, ciclo de outsourcing, contrataciones y las ventajas y desventajas de realizar externalizaciones en las unidades de información.

Palabras clave: Outsourcing, Externalización, Terciarización, Gestión de unidades de información, Contratos outsourcing.

\section{Title: Outsourcing in information units within or-}

\begin{abstract}
Outsourcing is a business management tool that contributes to the field of information by offering new ways for managing information units within organisations. Outsourcing requires acquiring an in-depth knowledge of the concept: its modes, cycles, contracting, new professional roles, as well as the advantages and disadvantages to the information unit that outsourcing can represent.
\end{abstract}

Keywords: Outsourcing, Information units, Information management, Outsourcing models, Outsourcing cycles, Contracts.

Lara Navarra, Pablo; Martínez Usero, José Ángel. “Outsourcing en las unidades de información de las organizaciones". En: El profesional de la información, 2002, mayo-junio, v. 11, n. 3, pp. 164-171.

\begin{abstract}
José Ángel Martínez Usero, es diplomado en biblioteconomía y documentación, licenciado en documentación y máster en sistemas de información. Trabaja como website manager para Calderdale MBC y como consultor de la licenciatura en documentación de la UOC (Universitat O berta de Catalunya).
\end{abstract}

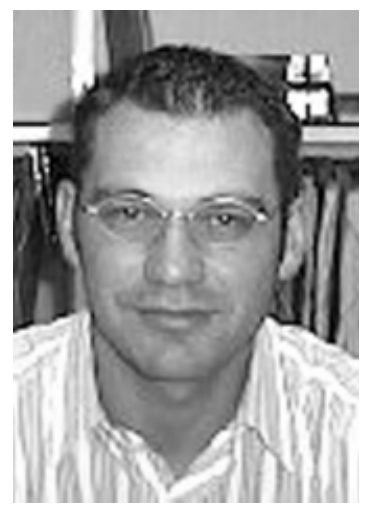

\section{Introducción}

Los retos tecnológicos, económicos y sociológicos de la sociedad de la información están demandando el desarrollo de nuevas formas de gestión en las organizaciones que hagan posible un mayor grado de flexibilidad para la innovación y fortalecimiento de la competitividad. De esta forma, se produce una ansiedad para la adecuación a las demandas de servicios y productos del mercado de la denominada globalización, pero que es difícil de alcanzar por uno mismo. Algunas de las causas argumentadas son: el factor económico, capacidad de asimilación de los cambios tecnológicos y necesidad de especialización. Esta situación conlleva que se deba recurrir a la unión o el apoyo de otras empresas mediante sistemas como la subcontratación, outsourcing, cooperativismo, joint venture, etc.
Entre todas las posibilidades nosotros queremos presentar el outsourcing o externalización.

Esta práctica surge en el mundo empresarial ante la necesidad de asimilación de las nuevas reglas del mercado marcadas por la capacidad de adaptación al cam-

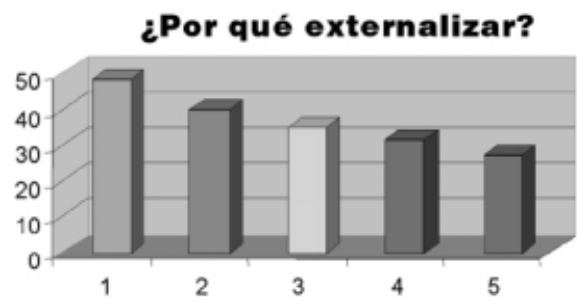

1. Reduc ción y control operacional de los costes 2.Rentabilizar las actividades nucleares de la organización 3.Ventajas de acceso a la competencia world-class 4. Reutilización de recursos para otras actividades 5. Disponer de recursos extemos a la organización 
bio. Estamos así ante una corriente opuesta y una superación de la filosofía promulgada desde la década de los 50: "bigger is better", que impulsaba la creación de grandes compañías que se expandían por todo el mundo alcanzando un alto nivel de diversificación y un control exhaustivo sobre todos los procesos ligados a la producción. Estas organizaciones son difíciles de gestionar por la implementación de modelos tradicionales basados en estructuras verticales y de gestión funcional jerárquicas, encontrándose muy burocratizadas y anquilosadas, impidiendo flexibilidad organizativa, creatividad empresarial, innovación y desarrollo de modelos competitivos.

\section{«El outsourcing ha evoluciona- do desde la gestión de activos para el ahorro de costes a la mejora de resultados comunes y mayor competitividad organi- zacional»}

En el campo de la información y documentación destaca el outsourcing implementado por las bibliotecas norteamericanas, la mayoría pertenecientes al sector universitario, que utilizan las externalizaciones para conseguir mayores beneficios —entiéndase como tales, entre otros, el aumento de la eficacia, de la eficiencia, reducción de costes, capacidad de innovación, etc.- para, de esta forma, poder alcanzar unos objetivos, ya sean de gestión administrativa como catalogaciones, circulación, etc. La razón principal por la cual la administración norteamericana tiende a ello son los altos costes que representa proporcionar la mayoría de los servicios.

\section{Qué entendemos por externalizar}

Debemos aclarar previamente que del término outsourcing no existe una definición única, debido a la variedad de los procesos de externalización. Si realizamos una revisión conceptual comprobamos la existencia de unos rasgos comunes basados en la transferen-

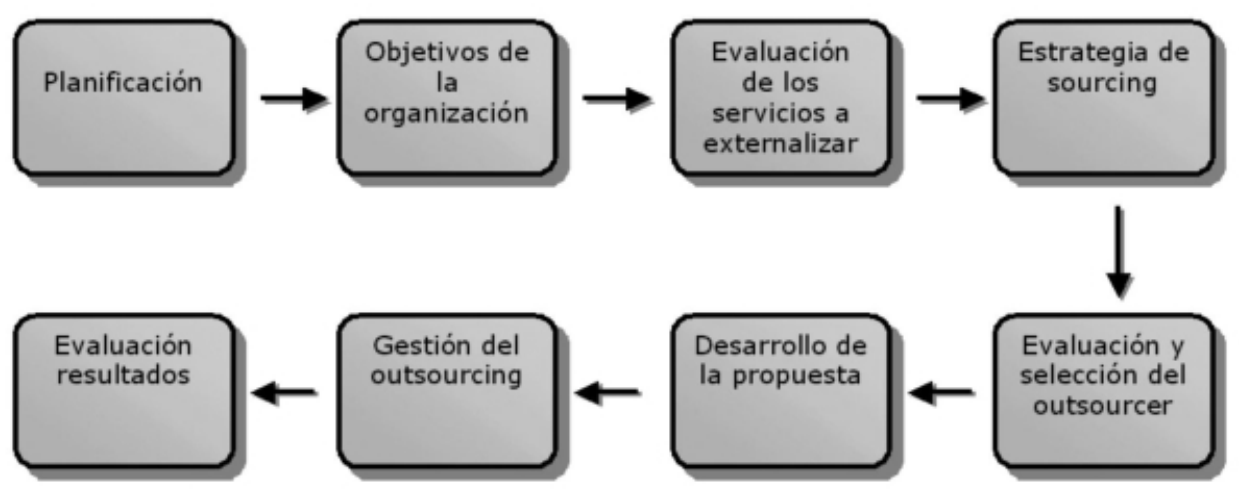

Figura 1

\section{Conceptos ligados al outsourcing}

Reducción de costes, especialización empresarial, mayor número de recursos, flexibilidad en la planificación, mayor adaptación en los desarrollos tecnológicos, reducción de burocracia, creatividad, mayor experiencia, mejor servicio al cliente.

cia, cesión, designación o externalización de áreas funcionales de una organización.

Los términos outsourcing, externalización o terciarización son empleados indistintamente. Se utilizan para definir una herramienta de gestión que designa un área o actividad de trabajo de nuestra organización para ser gestionada por otra empresa externa, denominada outsourcer (también podemos encontrar este término traducido como empresas prestadoras de servicios), especializada en este servicio demandado.

Outsourcing se puede definir también como la externalización de determinadas áreas funcionales, cediendo su gestión a sociedades de servicios externos. Handy (2000) define el término "como la externalización de aquellas funciones que no se consideran competencias nucleares de la organización" y emplea el concepto nuclear como el conjunto de actividades o funciones vitales de la misma.

\section{Outsourcing vs subc ontratación tradicional}

Antes de adentrarnos en la comparación de sus semejanzas y diferencias debemos tener presente que el primero es una modalidad de subcontratación. La subcontratación tradicional es definida someramente como el contrato civil o mercantil de arrendamiento de obra o de servicios. Desde una perspectiva jurídica no existe diferencia alguna entre la tipología de contratos de arrendamientos de obras y servicios y los de outsourcing. Por otro lado, las relaciones que se establecen entre las empresas impiden crear una subclase de subcontratos causado por la variedad de matizaciones que pueden ofrecer los contratos de externalización ${ }^{1}$. De esta manera, jurídicamente no existe diferencia entre outsourcing y subcontratación tradicional.

Las diferencias entre ambas se producen en el ámbito de la gestión organizacional, pues externalizar es sinónimo de cambio en la gestión con la finalidad de aunar es- 
fuerzos para que la empresa, tanto la prestataria como la prestadora del servicio, sean más competitivas. Por el contrario, la subcontratación tradicional refleja únicamente una vinculación contractual por concurso o designación directa por la cual, tras la realización de la obra o servicio, la relación establecida entre las empresas concluye.

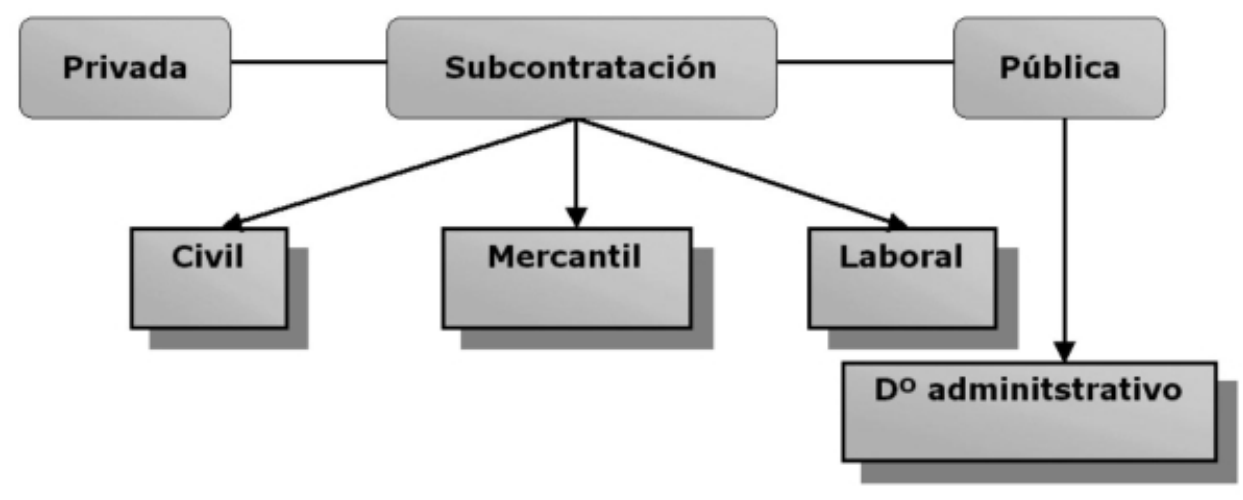

Figura 2

Outsourcing es un concepto cuyos fines económicos y causa de realización van más allá de un contrato de obras y servicios, implica esfuerzos mutuos para la consecución de objetivos establecidos entre ambas organizaciones, los beneficios repercuten en ambos contratantes, existiendo una estrecha vinculación de trabajo. Así pues, externalizar implica que la empresa externa (outsourcer), entra a formar parte de los activos de nuestra organización, naciendo entre ambas unos principios básicos de asociacionismo y mutua confianza, términos que destacábamos anteriormente. La transferencia de activos debe sentar las bases de una relación como si de socios se tratara y no de una mera relación jurídica cliente-proveedor.

\section{«No existe una definición única de outsourcing debido a la di- versidad de procesos que in- cluye»}

Existen diferentes modalidades de outsourcing:

-Total: comprendería la externalización completa de una actividad o función de una o más unidades de la organización. oficinas.

-Parcial: se lleva a cabo por regiones, sucursales,

- Selectivo: sólo implica las funciones o actividades menos importantes que no aportan un valor añadido a la organización, pero que su gestión impide un de-

Gartner Group Dataquest considera que para el año 2002 el $85 \%$ de las empresas europeas adoptarán estrategias de outsourcing selectivo como mecanismo de acceso a recursos de tecnologías de la información. Las tendencias en Europa se están expandiendo más allá de la gestión de infraestructuras y se orientan hacia la creación de valor en la organización.

sarrollo adecuado de las actividades vitales de la empresa.

Existen dos variantes de las formas de externalización expuestas anteriormente:

-Insourcing: conlleva remodelar los departamentos o unidades internas de la organización convirtiéndolos en outsourcers de la propia empresa.

-Cosourcing: es un proceso mixto, una combinación de algunas de las modalidades outsourcing junto al insourcing.

\section{Outsourcing en unidades de información}

1. Qué se entiende por externalización documental en España. En la práctica comenzó ligada al conjunto de servicios y productos de empresas dedicadas a la custodia de documentos y al principio se excluyeron de esa denominación otros servicios documentales.

Bajo esta concepción limitada de externalización se entendía pues el outsourcing documental como el ahorro de espacio y control del exceso de documentación generada por las organizaciones. Generalmente, estas empresas se caracterizan por ofrecer un software de gestión, un tratamiento especializado de la documentación y una custodia con garantías de confidencialidad y seguridad. Con estos servicios pretenden librar a las unidades de información de la carga que supone el almacenamiento y gestión de ingentes cantidades de documentos. El conjunto de servicios ofertado lo podemos estructurar en los siguientes apartados:

-Procesos técnicos: descripción del archivo, identificación de tareas archivísticas, criterios de ordenación y clasificación, junto a las propuestas de mejoras y actuaciones inmediatas.

-Análisis de costes: mano de obra, copia de documentos, distribución, infraestructura y material.

—Viabilidad y mejora de la externalización: aumento de la productividad en la empresa, reactivación 
de actividades básicas, reestructuración de personal, calidad en el acceso a la información, seguridad en la implantación, personal especializado, etc.

Estos son los servicios y productos entendidos por externalización documental, pero sólo son una muestra de actividades que se pueden realizar en las unidades de información. La diversidad de tareas susceptibles de llevarse a cabo obliga a una revisión del concepto de outsourcing documental, de manera que puedan integrarse las diversas casuísticas y peculiaridades de los centros españoles, yendo más allá de la custodia de documentos.

\section{«Desde una perspectiva jurídi- ca no hay diferencia entre la ti- pología de contratos de arren- damientos de obras y servicios y los de outsourcing»}

En nuestra opinión, la tarea de producir una definición precisa y actualizada de este concepto debería surgir de las instituciones y asociaciones profesionales del ámbito de la gestión de información. De hecho, una definición corporativa podría ayudar a promover estas actividades en las unidades de información, a la vez que favorecer el surgimiento de nuevas tareas y roles profesionales.

2. Ciclo de outsourcing en unidades de información. No se encuentra unificado pero sí podemos establecer unos pasos básicos que acrecentarán su complejidad según la modalidad de externalización seleccionada, que son los reflejados en la figura 1. Este ciclo de actividad en una unidad de información ha sido reunido en 4 grupos de acción, que son:

-Estudiar el plan estratégico y de marketing de la unidad de información.

—Análisis del outsourcer.

-Contratación: contactos y presentación del contrato.

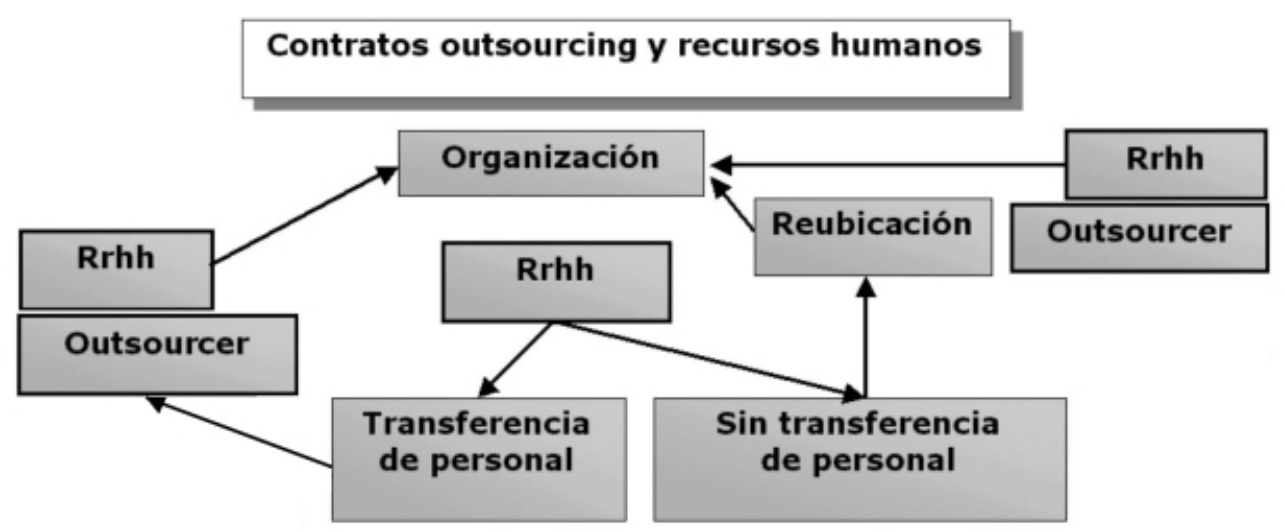

Figura 3

\section{0 razones para externalizar}

- Reducción y control operacional de los costes.

- Rentabilizar las actividades nucleares de la organización.

- Ventajas de acceso a la competencia worldclass.

- Reutilización de recursos para otras actividades.

- Disponer de recursos externos a la organización.

- Acelerar los beneficios de reingeniería.

- Gestión de actividades dificultosas.

- Disponer de capital social.

- Compartir riesgos.

- Inyección de capital.

Fuente: Outsourcing Institute M embership, 1998. servicio.

—Establecer los indicadores para la evaluación del

\section{a. Objetivos e identificación de tareas}

Estudiar el plan estratégico y de marketing de la organización y ver cuáles son las actividades y funciones a externalizar mediante un estudio de viabilidad. Esta tarea nos ayudará tanto a ver los objetivos y tareas nucleares de la empresa como los servicios y productos necesarios para satisfacer los objetivos marcados. Para su desarrollo podemos apoyarnos en el análisis dafo (debilidades, amenazas, fortalezas y oportunidades). La asociación Librarians in the 21 st century ${ }^{2}$ establece que las tareas que pueden ser objeto de externalización son: catalogación, indización, búsqueda de información y apoyo a la investigación, desarrollo web, archivo y custodia, servicios técnicos y gestión integral de la organización.

Este listado establece un conjunto muy heterogéneo de actividades que abarca la mayoría de los trabajos que se llevan a cabo en las unidades de información que implica el concepto de la gestión integral de dichos centros. De hecho, y como ejemplo, las bibliotecas han seguido las tendencias de negocio tradicionales $\mathrm{y}$ con el tiempo han ido ampliando las posibilidades de outsourcing desde la 
mera catalogación hasta casi la totalidad de tareas bibliotecarias.

\section{b. Estudio de la oferta}

Análisis de las empresas que pueden desarrollar las tareas a externalizar. Antes de la selección se diseñarán los puntos a estudiar para evaluar la elección del outsourcer. Algunos de estos indicadores pueden ser los Factores para la elección de la empresa externa del estudio realizado por el Outsourcing Institute , $^{3}$ donde se presentan en orden de importancia otorgada al outsourcer para la ejecución de la tarea o actividad:

- Compromiso de calidad.

-Precio.

—Referencias de otros clientes. bles.

—Términos del contrato flexi-

—Libertad de recursos.

- Capacidad de aportar valor añadido.

-Relaciones actuales.

-Ubicación.

-Otros.

Las empresas externalizadoras en gestión de información han evolucionado para responder a las nuevas necesidades de las unidades de información. Si atendemos al estudio The impact of outsourcing and privatization on library services and management ${ }^{4}$, en el apéndice $\mathrm{E}$, se proporciona una lista de empresas externalizadoras de catalogación, aunque en realidad lo que ofrecen son servicios integrales de outsourcing para bibliotecas. De forma similar, en las Librarian's yellow pages 5 (EUA), bajo el epígrafe de servicios de outsourcing, aparecen sólo 3 empresas y se nos remite a otras entradas como "cataloging services", "document imaging and scanning" o "information brokers". Por tanto, en el ámbito bibliotecario es un término bastante nuevo al que las bibliotecas tendrán que acostumbrarse en un futuro muy próximo y que deberán concebir como un apoyo para la realización de aque- llas tareas que no tienen capacidad de realizar por sí mismas o con los niveles de calidad mínimos establecidos.

En la tabla 1 presentamos un conjunto de empresas que ofrecen estos servicios en nuestro país, sin pretender ser exhaustivos sino meramente representativos.

\section{c. Contratación}

Contactos y presentación del primer borrador de contrato con las especificaciones de las tareas a externalizar. No existe un contrato estándar para el outsourcing, cada uno va a depender de los tipos e implicaciones de acuerdos que se realicen, de igual manera

\begin{tabular}{|c|c|c|}
\hline Azertia & http://www.azertia.es/ & $\begin{array}{l}\text { Servicios integrales relacionados } \\
\text { con las tecnologias de la } \\
\text { información, gestión } \\
\text { bibliotecas, integración y gestión } \\
\text { documental con ERPs y CRMs, } \\
\text { outsourcing de tratamiento } \\
\text { documental. }\end{array}$ \\
\hline Doc6 & http://www.doc6.es & $\begin{array}{l}\text { Gestión del conocimiento, } \\
\text { consultoria, formación, gestión } \\
\text { documental, automatización, } \\
\text { edición electrónica, bases de } \\
\text { datos. }\end{array}$ \\
\hline $\begin{array}{l}\text { Sistemas } \\
\text { Documentales }\end{array}$ & http://www.sisdoc.es/ & $\begin{array}{l}\text { Gestión documental, publicación } \\
\text { en internet/intranet, bases de } \\
\text { datos. }\end{array}$ \\
\hline Infodoc & http://www.infodoc.es & $\begin{array}{l}\text { Servicios } \\
\text { mantenimientos: } \\
\text { hardware, alquiler, outsourcing. }\end{array}$ \\
\hline Scanbit & http://www.scanbit.net & $\begin{array}{l}\text { Organización de unidades de } \\
\text { información, } \\
\text { digitalización, procesos masiónos, } \\
\text { consultoria, outsourcing, ASP } \\
\text { (application service providing). }\end{array}$ \\
\hline Recall & http://www.recall.com/span ish/index.htm/ & $\begin{array}{l}\text { Organización de archivos y centros } \\
\text { de documentación, custodia } \\
\text { externa de archivos, digitalización } \\
\text { de documentos, microfilm, base de } \\
\text { datos. }\end{array}$ \\
\hline $\begin{array}{l}\text { Databox } \\
\text { Andalucia S.A. }\end{array}$ & $\begin{array}{l}\text { http://www.ajecien.com/datab.htm/\#Punt } \\
\text { o\%20de\%20fijación31759 }\end{array}$ & $\begin{array}{l}\text { Gestión, organización y custodia } \\
\text { externa de archivos y fondos } \\
\text { documentales, informatización y } \\
\text { digitalización de archivos, } \\
\text { consultoría documental, formación } \\
\text { en gestión documental. }\end{array}$ \\
\hline Eprom & http://www.epromsl.es & $\begin{array}{l}\text { Sistemas de archivo y gestión } \\
\text { electrónica de documentos: GED, } \\
\text { organización de archivos, } \\
\text { digitalización y clasificación de la } \\
\text { documentación, custodia de la } \\
\text { documentación. }\end{array}$ \\
\hline MDA Archivos & http://www.mdaarchivos.es & $\begin{array}{l}\text { Organización, inventariado e } \\
\text { informatización de archivos, } \\
\text { actualización y racionalización de } \\
\text { archivos, inventariado y control } \\
\text { informatizado de mobiliario y } \\
\text { bibliotecas, custodia y gestión de } \\
\text { copias de seguridad de soportes } \\
\text { informáticos. }\end{array}$ \\
\hline $\begin{array}{l}\text { Zona Franca } \\
\text { de Cádiz }\end{array}$ & http://www.zonafrancacadiz.com & $\begin{array}{l}\text { Depósito y custodia de archivos, } \\
\text { análisis documental, digitalización, } \\
\text { expurgos, almacenaje de impresos } \\
\text { normalizados. }\end{array}$ \\
\hline Adea & http://www.adea.es & $\begin{array}{l}\text { Gestión y custodia de archivos } \\
\text { tanto activos como pasivos, centro } \\
\text { de outsourcing integral, insourcing } \\
\text { documental. }\end{array}$ \\
\hline
\end{tabular}




\title{
Complete su colección de IWE/EPI
}

Deseo recibir las siguientes publicaciones:

$\square$ _-_ ejemplares de la Bibliografía IWE/EPI 1992-2001 por correo postal impresos en papel (gratis).

$\square$ la Bibliografía IWE/EPI 1992-2001 por correo electrónico en formato PDF (gratis).

$\square$ _-- álbumes de los 2 co-roms con el texto íntegro de los primeros 100 números de la revista a 30 euros cada álbum (1 álbum es gratis para los suscriptores).

$\square$ _-- ejemplares de los siguientes números atrasados de la revista (por favor, envíenme presupuesto).

Nombre:

Institución:.

Dpto.:

N IF institucional:

Dirección:

Código postal:

Ciudad:

País:

Teléfono:

Fax:

Correo-e:

Envíe este boletín por correo postal, fax o correo electrónico, a esta dirección:

\author{
Swets Blackwell \\ Caspe, 46 \\ 08010 Barcelona \\ España \\ Tel.: +34-932 701 144; fax: 932701145 \\ mnzang@es.swetsblackwell.com
}

que no hay una legislación para regular este tipo de actividad. Brevemente, podemos establecer que jurídicamente los contratos outsourcing están amparados bajo el ámbito civil, mercantil y laboral ${ }^{6}$. Cuando introducimos las relaciones outsourcer con la Administración Pública entraría en juego además el derecho administrativo (figura 2).

En el momento de la formalización, podemos derivar la actividad al outsourcer sin involucrar personal de nuestra organización o transferirla junto con el grupo humano que conforma el departamento que externalizamos. Esta última casuística presenta uno de los mayores escollos en los contratos de outsourcing. La legislación no regula claramente cómo se ha de realizar esta transferencia, y aún en menor medida cuando en los contratos de externalización entran en juego las administraciones públicas. Algunas consultoras indican que el proceso más factible para poder transferir personal de la Administración del Estado bajo un contrato outsourcing es la creación de una nueva organización entre el outsourcer y la Administración (figura 3).

\section{«Externalizar implica que la empresa externa (outsourcer) entra a formar parte de los acti- vos de nuestra organización»}

Su adopción por parte de las administraciones públicas está amparada con la aprobación y modificación del apartado 3 del Artículo 196, definición de contratos de servicios, y el apartado número 5 del Artículo 198, sobre duración de los contratos de servicios, comprendidos en la Ley de Contratos de las Administraciones Públicas.

En el caso del outsourcing, se trata de un contrato atípico por no tener regulación alguna y no está sujeto a formalidad entre el empresario y el outsourcer (que puede ser una persona física o jurídica). Otras de sus 
características son su carácter oneroso, consensual, conmutativo y bilateral.

\section{«Las bibliotecas han ido am- pliando las posibilidades de outsourcing desde la mera ca- talogación hasta la casi totali- dad de las tareas biblioteca- rias»}

Independientemente del contrato que escojamos, debe predominar la claridad y transparencia con un carácter asociativo. Además, debemos conocer una serie de puntos básicos antes de formalizarlo. Se ha de contemplar:

- Claridad en la definición de las actividades y funciones que van a ser externalizadas.

-Especificación de los servicios y condiciones a llevar a cabo.

—Designación clara y concisa de obligaciones.

-Establecimiento mutuo de los diferentes procesos de seguimiento y control durante la prestación del servicio.

-Acceso a información interna por parte del proveedor.

-Procedimientos para la resolución de conflictos.

-Designación de los canales de comunicación y de los interlocutores entre ambas partes.

-Especificación de sanciones por incumplimiento de contrato y, del mismo modo, incentivos que se aplicarán por los servicios prestados por encima de las condiciones acordadas.

-Establecimiento de una cláusula de rescisión del contrato, con todas las condiciones bien detalladas para que no dé posibilidad a malentendidos.

Es importante contar con expertos externos para que puedan ayudar y asesorar durante la fase de acuerdo.

\section{d. Procedimiento de evaluación}

Establecer los indicadores para la valoración del servicio junto a los calendarios de entregas. La conclusión de la lectura de evaluación nos debería llevar a un cuadro que reflejará las siguientes ventajas e inconvenientes:

1. Aspectos que en un primera evaluación deberían ser positivos al realizar outsourcing en unidades de información.

-Reducción de tiempos. El outsourcer es experto y está especializado en el tipo de actividad externalizada.
-Disminución de los costes. Si por ejemplo externalizamos la catalogación de nuestra unidad de información, realizaremos un estudio de cuánto nos cuestan las catalogaciones, ya sean capturadas en red, modificación de registros o de nueva creación para, más tarde, poder compararlos con los precios de la empresa externa. Además, debemos fijarnos en la reducción de la relación coste-eficacia.

-Mayor control en los contenidos.

-Reestructuración del personal en otras funciones o actividades descuidadas.

—Reducción de burocracia administrativa.

-Rápida adaptación a los cambios tecnológicos que es necesario realizar en el sistema de información ya sea la biblioteca, archivo o centro de documentación. Un ejemplo podría ser la migración a otros sistemas.

-Información detallada mediante informes, dossieres, etc., de la evolución del trabajo.

2. En cuanto a los aspectos que en un primer análisis podrían resultar negativos en su aplicación:

-Coordinación entre equipos de trabajo.

-Costes anticipados de puesta a punto para poder realizar el proyecto.

- Puntualidad en la entrega en cada fase del proyecto.

- Transferencia de personal a otros departamentos o a la empresa outsourcer como parte del contrato de externalización.

-Cuestiones técnicas y legales para la contratación.

\section{Perfiles profesionales}

Hemos hablado brevemente sobre qué es outsourcing, cómo se desarrolla, qué implica y de las externalizaciones en unidades de información; pero también se ha de tratar el perfil del personal responsable de la ejecución de este trabajo en las empresas externalizadoras. Sus características ideales serían las de un profesional de la información capaz de afrontar todas las tareas que una unidad de información necesite externalizar.

La formación de este profesional en nuestro país ha sido muy heterogénea. Al principio se hacía a sí mismo mediante la práctica del ejercicio profesional; en los últimos años, se ha venido formando en la universidad, donde adquiere un nivel de conocimientos que le permiten el desarrollo de su trabajo en cualquier unidad de información. Esto es, se trata de un nuevo 
profesional capaz de liderar una empresa outsourcer que proporcione servicios a esos centros, capacitado para realizar la gestión integral de la información en las organizaciones - desde la custodia de documentos, todo tipo de tratamiento documental físico o virtual, el desarrollo de herramientas para la gestión de información, etc. (véase la tabla de servicios de las empresas outsourcers en España)-.

Podemos observar que la profesión avanza hacia la figura de un profesional polivalente, multidisciplinar e interdisciplinar. No es suficiente poseer conocimientos de diferentes disciplinas, lo importante es saber cómo integrarlas bajo un mismo y único conocimiento.

\section{Conclusiones}

-El outsourcing tiende hacia la construcción de una relación basada en la asociación, en compartir riesgos y en la búsqueda de resultados comunes más allá de la reducción de costes.

- Realizar externalizaciones no será en muchos casos la solución a los problemas planteados en las unidades de información, pues simples subcontrataciones por el marco temporal o económico de la actividad resultan más rentables que establecer vínculos de outsourcing.

-Esta práctica tiene una serie de inconvenientes que pueden ser insalvables a la hora de realizarla: de carácter organizativo de la actividad, como el trasvase de personal, reorganización de actividades, coordinación de equipos, etc.; o por la naturaleza de la institución, siendo un ejemplo de ello la falta de flexibilidad para aplicarlo en el contexto de las administraciones públicas.

-El outsourcing no conlleva, por sí solo, la mejora de productividad y rendimientos de las unidades de información. Se requiere un esfuerzo de adaptación y de trabajo común con otras organizaciones.

— La evaluación de la actividad externalizada debe tener unos valores positivos respecto a los costes (eficiencia) y tiempos y eficacia (calidad).

-Debemos saber cuáles son las funciones y actividades básicas o nucleares antes de llevar a cabo outsourcing y valorar si verdaderamente es viable.

- Hay que conocer bien las ventajas que ofrece a nuestra organización el outsourcer seleccionado.

-Es conveniente estudiar y establecer un contrato outsourcing con el mayor número de especificaciones posibles.

\section{Notas}

1. Cruz Villalón, Jesús. Outsourcing y relaciones laborales. Consultado en: 01-03-02.

http://www.lex.unict.it/dml-online/archivio/numero3/online/cruz.htm

2. Librarians in the 21st Century. Outsourcing. Consultado en: 01-03-02. http://istweb.syr.edu/21stcenlib/where/outsourcing.html

3. The outsourcing Institute. Consultado en: 01-03-02.

http://www.outsourcing.com

4. ALA. The impact of outsourcing and privatization on library services and management. Consultado en: 01-03-02.

http://www.ala.org/alaorg/ors/outsourcing/index.html

5. Librarian's yellow pages. Consultado en: 01-03-02.

http://www.librariansyellowpages.com

6. El contrato de outsourcing, en los ámbitos civil y mercantil, está regulado por el correspondiente contrato de arrendamientos de servicios o de obra, y por el Estatuto de los Trabajadores en materia de: contratas y subcontratas de obras y servicios, el Artículo 42; cesión de trabajadores, el Artículo 43; y la sucesión de empresa, el Artículo 44.

\section{Bibliografía}

Baker, Barry B. "Resource sharing: outsourcing and technical services". En: Technical services quarterly, 1998, v. 16, n. 2, pp. 35-45.

Balagué Mola, Núria. "L'externalització o la flexibilitat de la gestió". En: Item, 2001, n. 28, pp. 4-13.

Benaud, C. L.; Bordeianu, S. Outsourcing library operations in academic libraries: an overview of issues and outcomes. Colorado: Libraries Unlimited, 1998.

Bush, Carmel C.; Sasse, M.; Smith, P. "Toward a new world order: a survey of outsourcing capabilities of vendors for acquisitions, cataloging and collection development services". En: Library acquisitions: practice \& theory, 1994 , v. 18, n. 4 , pp. 397-414.

Fogerty, James E. "Archival brinkmanship: downsizing, outsourcing, and the records of corporate America". En: American archivist, 1997, v. 60 , pp. 44-55.

Garcia-Abril, Carlota. "Outsourcing documental”. En: Outsourcing, 1999, pp. 18-22.

Gidron, Gil. Nuevos modelos de gestión empresarial: el outsourcing de procesos de negocios. Madrid: Círculo de Empresarios, 1998.

Hirshon, Arnold. Outsourcing library technical services. New York: Neal-Schuman, 1996

Horestein, Bonnie. "Outsourcing copy cataloging at Adelphi University libraries". En: Cataloging \& classification quarterly, 1999, v. 28, n. 4, pp. 105-116.

Howarth, Lynne C. "The role of the paraprofesional in technical services in libraries”. En: Library trends, v. 46, n. 3, pp. 526-539.

Lara Navarra, Pablo; Martínez Usero, José Ángel. “Outsourcing documental: organización de futuro”. En: VII Jornadas españolas de documentación, 2000, pp. 49-55.

Ogburn, Joyce L. "An introduction to outsourcing”. En: Library acquisitions: practice \& theory, 1994, v. 18, n. 4, pp. 363-366.

Peso Navarro, Emilio. Manual de outsourcing informático: análisis y contratación. Madrid: Díaz de Santos, 2000.

Reuvid, Jonathan; Hinks, John. Managing business support services: strategies for outsourcing \& facilities management. London: Kogan Page, 2001.

Pablo Lara Navarra y José Ángel Martínez Usero, Universidad Oberta de Catalunya.

plara@uoc.edu

jmartinezu@uoc.edu 